\title{
Comparing Structures of Two Antimicrobial Peptides Using a Self-Organized Mapping Approach
}

\author{
Faramarz Joodaki ${ }^{1}$, Preston Steele ${ }^{2}$, Lenore M. Martin ${ }^{3}$, and \\ Michael L. Greenfield ${ }^{1}$ \\ ${ }^{I}$ Department of Chemical Engineering; ${ }^{2}$ Department of Electrical, Computer, and Biomedical Engineering; \\ ${ }^{3}$ Cell and Molecular Biology, University of Rhode Island, Kingston, RI, 02881, USA
}

\section{Introduction}

Antimicrobial peptides (AMP) show future potential as drugs against diverse kinds of bacteria [1]. The AMP mechanism of action is specified by its structure, which continues to change (folding process) during its attack on the membrane of bacteria [2]. Hence, the structure of AMPs has a crucial role in their functionality. Although this bacterial attack mechanism has been studied with experiments and some models have been hypothesized to explain it, a full understanding of the details has not been achieved [3-5]. Molecular modeling is a promising tool that can contribute to the better understanding of peptide structures and mechanisms of interaction with cell membranes [5].

A wide range of low energy conformations is usually obtained during molecular modeling, and each conformation may have different structural characteristics. Interpreting this huge set of simulation results from biological and biochemical perspectives is challenging because of the numerous small differences among conformations. The k-means clustering algorithm is a useful strategy to classify modeling data via physical and biological descriptors into different semi-homogeneous clusters on the basis of the distance from each data point to the center of each group. Each cluster is a region with a high density of data points which represent peptide conformations [6].

In this work, the structures of novel hybrid AMPs LM7-1 and LM7-2 [7], which were designed using combinations of pieces of naturally occurring AMPs, are investigated. These two AMPs differ in sequence at the $15^{\text {th }}$ residue: glycine in LM7-1 compared to lysine in LM7-2. These AMPs do not adopt an amphiphilic helical shape until they contact a bacterial membrane [7]. Here, molecular modeling and data clustering are used to compare the structures of these two AMPs to study how this small sequence difference affects their range of accessible conformations and the ability to attain helical structures.

\section{Results and Discussion}

Monte Carlo (MC) simulation as a molecular modeling tool was applied using the Towhee program [8] to sample from low energy structures of LM7-1 at room temperature (298K) and of LM7-2 at both room temperature $(298 \mathrm{~K})$ and biological temperature $(310 \mathrm{~K})$. Charmm 27 force field was used for calculating the potential energy. Towhee implements MC moves that include configurational bias for conformation rearrangement, pivots along dihedral angles, and atom displacements. It attempts random movements in each MC step to sample the potential energy surface of each AMP.

Figure 1 and 2 respectively show the changes in the total energy and the end-to-end distances of the two simulated AMP molecules after 800,000 cycles of MC steps. The steady fluctuations in the potential energy (Figure 1) suggest that each peptide molecule has equilibrated during the simulation. This is only one measure, however; the end-to-end distance during these three simulations continues to decrease and has not yet equilibrated at the end of the simulation. Since the conformations for these three simulations were still evolving at the end of 800,000 steps (Figure 2), equilibration cannot be determined using energy monitoring alone. The current three simulations sample among multiple AMP configurations of similar potential energies. However it is clear that continuing the MC simulations further to reach steady fluctuations in all variables, i.e. equilibration, is required. Further research to pursue such calculations is ongoing. 


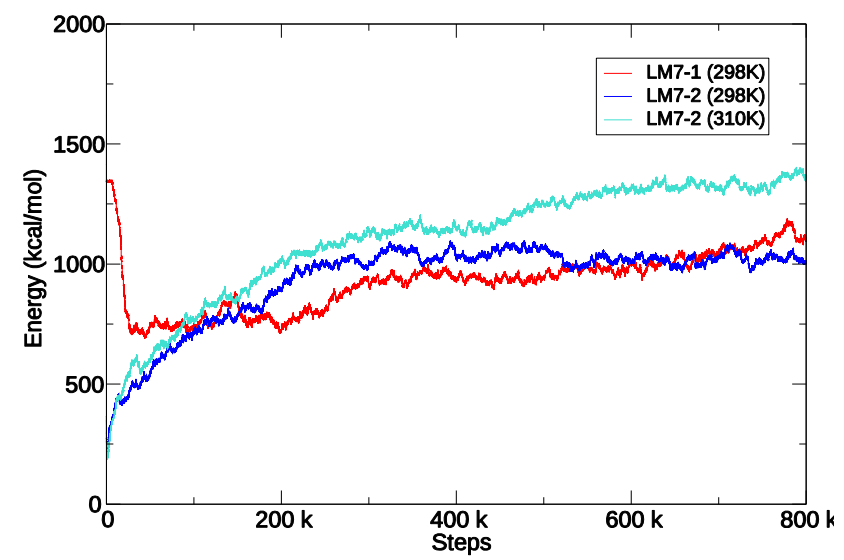

Fig. 1. Total energy distance of LM7-1 (at 298K) and LM7-2 (at 298 and 310K) during the MC simulation.

To facilitate the biological investigation of the current set of simulated peptide conformations, the Clustering Peptides and Proteins Software (CPP software) with graphic user interface (GUI) was developed using MATLAB. Just using the positions of all atoms (information found in the pdb files from the MC simulations), the CPP software calculates and displays some important physical and biological characteristics of the antimicrobial peptides: potential energies; propensities for specific secondary structures such as extended chains, alpha helices, pi helices, parallel and anti-parallel beta sheets; radii of gyration; end-to-end distances; and such shape parameters as acylindricity and asphericity. The CPP software then applies the K-means clustering algorithm based on a particular set of three calculated characteristics chosen by the user to aggregate closely related conformations from the simulation results and plot them on the (Self) organized 3-D map.

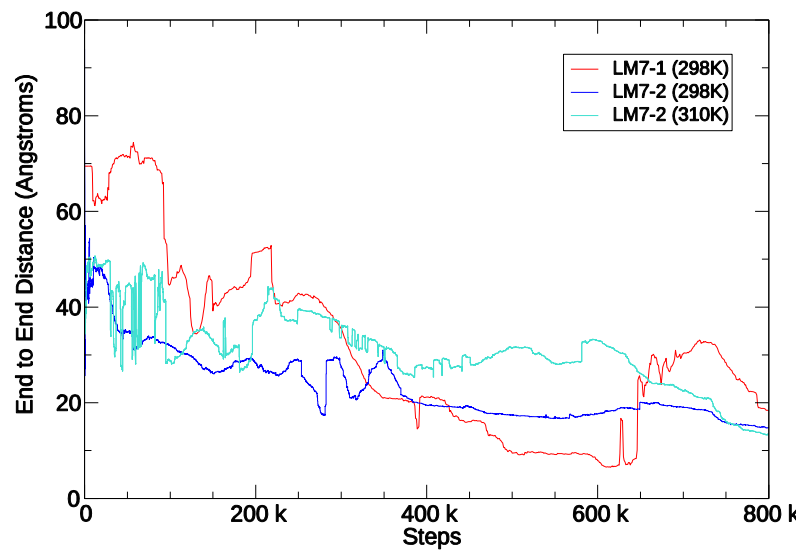

Fig. 2. End-to-end distance of LM7-1 (at 298K) and LM7-2 (at 298 and 310K) during the MC simulation.

The CPP software was fist applied to 1,000 MC simulation conformations (between 35,000 to 800,000 steps at $298 \mathrm{~K}$ ) of LM7-1 and then to 8,000 MC simulation conformations (between 0 to 800,000 steps at $310 \mathrm{~K}$ ) of LM7-2. Figures 3 and 4, respectively, show examples of k-clustering results generated by the CPP software with radius of gyration $\left(r_{g}\right)$, asphericity (bshape) and energy as the chosen three parameters. Although the initial structures for LM7-1 and LM7-2 during each MC simulation differ, the red clusters in both figures have some similar characteristics: the center point of the red cluster in figure 3 is $r_{g}=10.0 \AA$, bshape $=0.09$ and energy $=1375 \mathrm{kcal} / \mathrm{mol}$, while in Figure $4 \mathrm{r}_{\mathrm{g}}=9.28 \AA$, 
bshape $=0.33$ and energy $=1316 \mathrm{kcal} / \mathrm{mol}$. The energy and the radius of gyration for these clusters of conformations of the two peptides are quite similar, while the shape characteristics are very different. The LM7-1 structure has a more spherical shape, while LM7-2 tends to have more of a coin-shape. The cause of this shape difference and how it will tend to change in the presence of a lipid membrane are both interesting questions that are currently under investigation. Additional clusters in the LM7-1 simulation results show an overall trend from an initially extended chain conformation to a more compact conformation as the simulation proceeds. Some clusters for LM7-2 show much lower energies than for LM7-1. Figure 1 indicates that these low energy structures arise early in the simulation, near an initial $\alpha$-helix conformation. The spontaneous rise in energy observed in Figure 1 from 0 to 200,000 steps indicates that this initial low-energy region is shallow compared to the thermal energy $k_{\mathrm{B}} T$, at least without a hydrophilic or aqueous environment surrounding the peptide.

Several interesting observations can be obtained from analysis of the clustering based on groups of three chosen sets of diverse parameters over the course of the molecular modeling simulations. We are continuing to improve the CPP software and the next version will be open source and freely distributed. Adding the capability to choose additional meaningful biological parameters, such as hydrophobicity, is also currently under development.

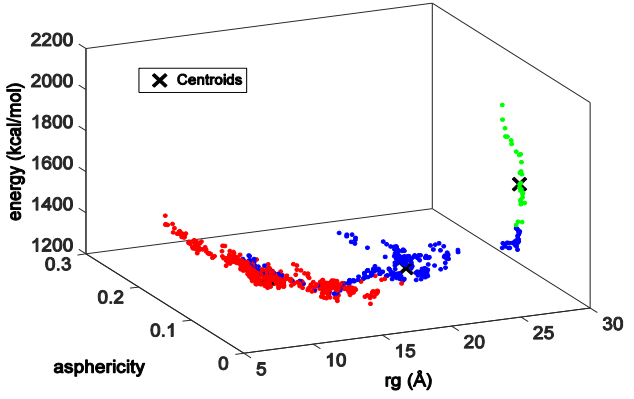

Fig. 3. Clustering results for 1000 conformations of LM7-1 (between 35,000800,000 steps).

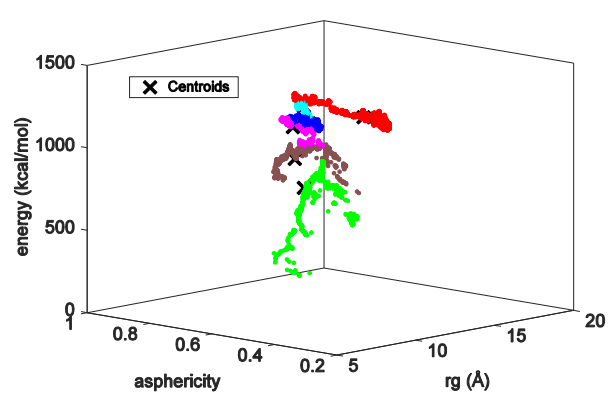

Fig. 4. Clustering results for 8000 conformations of LM7-2 (between 0-800,000 steps).

\section{Acknowledgments}

We thank the students in BCH-CSC522 Bioinformatics for helpful discussions and criticism. We thank Professor Jean-Yves Hervé for suggesting the k-means clustering algorithm, and Professors Martin and Greenfield for guiding stimulating discussion about the relevant propensities that could be plotted to better understand the changes in the peptide conformations and their effects during simulation.

\section{References}

1. Jenssen, H., Hamill, P., Hancock, R.E.W. Clin. Microbiol. Rev. 19, 491-511 (2006), http://dx.doi.org/10.1128/CMR.00056-05

2. Powers, J.S., Hancock, R.E.W. Peptides 24, 1681-1691 (2003),

http://dx.doi.org/10.1016/i.peptides.2003.08.023

3. Leontiadou, H., Mark, A.E., Marrink, S.J. J. Am. Chem. Soc. 128, 12156-2161 (2006), http://dx.doi.org/10.1021/ja062927q

4. Wang, K.F., Nagarajan, R. Camesano, T.A. Biophys. Chem. 196, 53-67 (2015), http://dx.doi.org/10.1016/j.bpc.2014.09.003

5. Wang, Y., Zhao, T. Wei, D., Strandberg, E., Ulrich, A.S., Ulmschneider, J.P. Biochim. Biophys. Acta -

Biomembr. 1838, 2280-2288 (2014), http://dx.doi.org/10.1016/j.bbamem.2014.04.009

6. Likas, A. Vlassis, N., Verbeek, J.J. Pattern Recognit. 36, 451-461 (2003).

7. LM7-1 and LM7-2 peptides were designed and synthesized by L. M. Martin and D. Ryder, University of Rhode Island, Dept. of Cell Mol. Biol.

8. Martin, M.G. "MCCCS Towhee: a tool for Monte Carlo molecular simulation," Mol. Simul. 39, 212-1222 (2013), http://dx.doi.org/10.1080/08927022.2013.828208 